\title{
Complex groundwater flow systems as traveling agent models
}

Analyzing field data from pumping tests, we show that as with many other natural phenomena, groundwater flow exhibits a complex dynamics described by $1 / f$ power spectrum. This result is theoretically studied within an agent perspective. Using a traveling agent model, we prove that this statistical behavior emerges when the medium is complex. Some heuristic reasoning is provided to justify both spatial and dynamic complexity, as the result of the superposition of an infinite number of stochastic processes. Even more, we show that this implies that non-Kolmogorovian probability is needed for its study, and provide a set of new partial differential equations for groundwater flow. 
3 1Former: Posgrado en Ciencias de la Tierra, Instituto de Geología, Universidad Nacional Autónoma de México, 4 Circuito Escolar, Cd. Universitaria México D.F.

5 2Currently: Theoretical Astrophysics, Instituto de Astronomía, Universidad Nacional Autónoma de México,

6 Circuito Escolar, Cd. Universitaria México D.F. olopez@astro.unam.mx.

7 3IIMAS, Universidad Nacional Autónoma de México, Circuito Escolar, Cd. Universitaria México D.F.

8 4Instituto de Geología, Universidad Nacional Autónoma de México, Circuito Escolar, Cd. Universitaria México D.F.

9 5Instituto de Geofísica, Universidad Nacional Autónoma de México, Circuito Escolar, Cd. Universitaria México D.F.

10 6Posgrado en Ciencias Biológicas, Facultad de Ciencias, Universidad Nacional Autónoma de México, Circuito 11 Escolar, Cd. Universitaria México D.F.

12 7Departamento de Matemáticas, Universidad Nacional Autónoma de México, Circuito Escolar, Cd. Universitaria 13 México D.F.

14 Keywords: Hydrogeology, Complex Systems, 1/f Noise, Quantum Game Theory, 15 Spatially Extended Games.

17 Pink or $1 / \mathrm{f}$ noise (sometimes also called Flicker noise) is a signal or process with a 18 frequency spectrum such that the power spectral density is inversely proportional to the 
19 frequency (Montroll and Shlesinger 1982; Downey 2012). This statistical behavior appears in 20 such diverse phenomena as Quantum Mechanics (Bohigas et al. 1984; Faleiro et al. 2006; Haq et

21 al. 1982; Relanyo et al. 2002), Biology (Cavagna et al. 2009; Buhl et al. 2006; Boyer and López-

22 Corona 2009), Medicine (Goldberger 2002), Astronomy and many other fields (Press 1978).

23 Recently the universality of $1 / \mathrm{f}$ noise has been related with manifestation of weak ergodicity

24 breaking (Niemann et al. 2013) and with statistical phase transition (López-Corona et al. 2013).

25 In Geosciences the idea of self-organized criticality (SOC) associated with $1 / \mathrm{f}$ power

26 spectrum showed to be important for example in modeling seismicity (Bak et al. 1987; Bak and

27 Tang 1989; Bak and Chen 1991; Sornette et al. 1989). The basic idea of SOC is that large

28 (spatially extended) interactive systems evolve towards a state in which a minor new event can

29 have dramatic consequences. In seismicity this means that earthquakes contribute to organize the

30 lithosphere both in space and time (Sornette et al. 1990). In this context, the lithosphere may be

31 understood as an unstable and non-linear system made of hierarchy of interacting blocks and in

32 which dynamics has a characteristic $1 / \mathrm{f}$ signal (Keilis-Botok 1990).

33 A particular active research field in Geoscience is the study of groundwater, which may be

34 considered as a complex dynamic system characterized by non-stationary input (recharge), output

35 (discharge), and response (groundwater levels). For example, groundwater levels in unconfined

36 aquifers never reach steady state and may vary over multiple spatial and temporal scales showing

37 fractal scaling characterized by inverse power law spectra (Zhang and Schilling, 2004 ; Jianting

38 et al. 2012). For a review on grounwater transport see Dentz et al. (2012).

39 Spectral analysis has proven to be a powerful analytical tool for the study of variations in

40 hydrologic processes. Ever since Gelhar (1974) studied temporal variations of groundwater levels

41 for the first time with spectral analysis, it has been widely used. Spectral densities have been used

42 in the study of: the earth tides effect on water level fluctuations (Shih et al. 2000; Maréchal et al.

43 2002); temporal scaling in discharge (Tessier et al. 1996; Sauquet et al. 2008); scaling in 
44 hydraulic head and river base flow (Zhang and Schilling, 2004; Zhang and Li, 2005, 2006); water

45 quality variations in space and time domains (Duffy and Gelhar 1985; Duffy and Al-Hassan

46 1988; Kirchner et al. 2000; Schilling et al. 2009). Using the Detrended Fluctuation Analysis

47 (DFA) method, Zhongwei and You-Kuan (2007) have proved that groundwater levels exhibit a

$481 / \mathrm{f}$ behavior for large time scales.

49 The groundwater flow process may be considered as the motion of agents (water

50 particles) in a heterogeneous medium (Tranouez et al. 2001; Cortis and Knubdy 2006; Park et al.

51 2008). This problem is analogous to the model of traveling agents presented in (Boyer and

52 López-Corona 2009). In that model, the agents are frugivorous animals who feed on randomly

53 located vegetation patches, in a similar way to anomalously diffusing particles in a physical

54 context. The displacement patterns of a variety of animals as albatrosses, bumble-bees, primates,

55 gastropods, jackals, seals and sharks, among others (Viswanathan et al. 1999, Ramos-Fernández

56 et al. 2004, Seuront et al. 2007, Atkinson et al. 2002, Austin et al. 2004, Sims et al. 2008) involve

57 many spatio-temporal scales and are sometimes well described by Lévy walks. This is the case of

58 the traveling agents of the model referred to (Boyer and López-Corona 2009). A good review on

59 biological aspects of the subject may be found in Miramontes et al. (2012) and for Lévy process

60 see Shlesinger et al. (1982), Klafter et al. (1987) and Lomholt et al (2008).

61 The frequent occurrence of pink noise in a seemingly unrelated set of physical systems

62 has prompted an extensive search for common underlying physical principles (Miller et al. 1993).

63 In this paper we present a heuristic reasoning for the emergence of $1 / \mathrm{f}$ noise in groundwater and

64 propose a new set of groundwater equations for flow in complex media (see electronic 65 supplementary materials).

$66 \quad 1.1 \quad$ The traveling agent model

67 Let us consider a two-dimensional square domain of unit area with $\mathrm{N}$ fixed, point-like 68 food patches randomly and uniformly distributed. Each patch contains an amount of food k. 
Initially, an agent is located on a patch chosen at random. Then the following

70 deterministic foraging rules are iteratively applied at every time step:

71 (i) The agent located at patch i feeds on that patch, the fruit content decreasing by one 72 unit: $\mathrm{k}_{\mathrm{i}} \rightarrow \mathrm{k}_{\mathrm{i}}-1$.

73 (ii) If ki has reached the value 0 , the agent chooses another patch, $\mathrm{j}$, such that $\mathrm{k}_{\mathrm{j}} / \mathrm{d}_{\mathrm{ij}}$ is

74 maximal over all the allowed patches $\mathrm{j} \neq \mathrm{i}$ in the system, where $\mathrm{k}_{\mathrm{j}}$ is the food content of patch $\mathrm{j}$

75 and dij the Euclidean distance between patches $i$ and $j$. With this rule, the next visited patch (the

76 "best" patch) has large food content and/or is at a short distance from i. It was assumed that the 77 travel from $i$ to $j$ takes one time unit.

78 (iii) The agent does not revisit previously visited patches.

79 This model produces complex trajectories that have been studied in detail in refs. (Boyer 80 et al. 2006; Boyer et al. 2009) and discussed in connection with spider monkeys foraging patterns 81 observed in the field (Ramos-Fernández et al. 2004). Most interesting is the fact that when this 82 model is combined with a forest one, the coupled model exhibits self-organized criticality and 1/ 83 f power spectrum for biomass time series (Boyer and López-Corona 2009).

84 We propose that it is possible to use an equivalent model to study groundwater flow, 85 conceptualizing it as the motion of water particles (agents) in a hydrogeological medium. Assume the existence of a scale of support $\mathrm{w}$ where porous media properties can be

87 measured. This scale of support is kept constant and is small enough such that, at the scale of the

88 flow domain, w can be represented as a point-like quantity. Let us consider a two-dimensional 89 square domain of unit area with $\mathrm{N}$ fixed, point-like Hydrogeological Units (HU) randomly and 90 uniformly distributed. Each $\mathrm{HU}$ is characterized by its hydraulic flow potential, defined as $\mathrm{K}_{\mathrm{i}}=\mathrm{H}_{\mathrm{i}}$ $91 / \mathrm{R}_{\mathrm{i}}$, where $\mathrm{H}_{\mathrm{i}}$ and $\mathrm{R}_{\mathrm{i}}$ are hydraulic head and hydraulic resistivity at point $\mathrm{i}$, respectively. Thus $\mathrm{K}_{\mathrm{i}}$ 92 has units of time.

93 Initially, an agent (water particle) is located on a HU chosen at random. Then the 
94 following deterministic motion rules are iteratively applied at every time step:

95 (i') An agent located in a HU stays there for a dimensionless time $\mathrm{T}$ proportional to

$96 \mathrm{~K}_{\max } /(\mathrm{K}+\mathrm{a})$, where $\mathrm{K}_{\max }$ is the maximum hydraulic flow potential in the domain and a is an

97 arbitrary normalization constant such that $\mathrm{K}_{\max }>>$ a. For $\mathrm{K} \rightarrow 0$ then the waiting time is the

98 maximum possible; For $\mathrm{K} \rightarrow \mathrm{Kmax}$ then the waiting time is the minimum possible.

99

(ii') When an agent has spent $\mathrm{T}$ time in the $\mathrm{HU}_{\mathrm{i}}$, it chooses another $\mathrm{HU}_{\mathrm{j}}$, such that $\Delta \mathrm{K}_{\mathrm{ij}}$ /

$100 \mathrm{~d}_{\mathrm{ij}}$ is maximal over all the allowed $\mathrm{HU}(\mathrm{i} \neq \mathrm{j})$ in the domain, where $\Delta \mathrm{K}_{\mathrm{ij}} / \mathrm{d}_{\mathrm{ij}}$ is the hydraulic flow

101 potential difference between $\mathrm{HU}_{\mathrm{i}}$ and $\mathrm{j}$, and $\mathrm{d}_{\mathrm{ij}}$ is the Euclidean distance between points $\mathrm{i}$ and $\mathrm{j}$.

102 With this rule, the next visited HU has the largest hydraulic flow potential gradient. It is assumed 103 that the travel from $\mathrm{i}$ to $\mathrm{j}$ takes one time unit.

104 (iii') For a particular set of initial and boundary conditions, the agent does not revisit 105 previously visited HU.

106 With this set of rules, both models, biological and groundwater flow, have the same

107 statistical properties despite representing very different systems and then a direct analogy may be 108 considered.

109 This traveling agent model exhibits some remarkable properties. Let us define the 110 displacement of an agent $\mathrm{R}(\mathrm{t})=|\mathrm{R}(\mathrm{t}+\mathrm{t} 0)-\mathrm{R}(\mathrm{t} 0)|$ with $\mathrm{R}(\mathrm{t})$ is the location of the agent at time $\mathrm{t}$.

111 For analysis, averages werte taken over different and independent realizations. If the hydraulic

112 flow potential $\mathrm{K}$ follows an inverse power-law distribution $\mathrm{P}(\mathrm{K})=\mathrm{cK}^{-\beta}$, where $\mathrm{c}$ is an arbitrary

113 constant and $\beta$ is a coefficient that represents the medium homogeneity. When $\beta$ is large $(\beta>>1)$

114 the medium is very homogeneous, meaning that all HU have similar values of hydraulic flow 115 potential. On the contrary when $\beta$ is small $(\beta \sim 1)$ the medium is very heterogeneous, meaning that 116 HUs with high hydraulic flow potential are numerous. The intermediate case is when $\beta=3$ and 117 corresponds to a complex medium where HUs with high hydraulic flow potential are present but 
they are not so numerous.

119

120

121 are the result of systems which superimpose the transmission of many independent stochastic 122 signals.

123 With this in mind, we proceeded to investigate if the power spectrum of the agent's 124 motion follows a $1 / \mathrm{f}$ dynamics. We found a non-trivial relationship between the homogeneity 125 coefficient $\beta$, the motion of the traveling agent and the type of noise observed. These results 126 (summarized in Table 1) are new and differ from previous work since now the motion of the 127 agents is explicitly analyzed.

128 129

130

131

132

133

134

135

Table 1 Relation between media homogeneity coefficient $\beta$, type of medium, agent motion, and the noise type observed.

Figure 1 Power spectra for traveling agents with three values of homogeneity. (a) First column $\beta=2$, the medium is very inhomogeneous (disordered) and the signal is a white noise. (b) Second column $\beta=3$, the medium is complex and the signal is a pink noise. (c) Third column 5, the medium is very homogeneous (ordered) and the signal is a brown noise. Power Spectrum is taken as $\mathrm{S}(\mathrm{f}) \equiv \tilde{\mathrm{R}}(\mathrm{f}) \tilde{\mathrm{R}}(-\mathrm{f})$, where $\tilde{\mathrm{R}}(\mathrm{f})$ is the Fourier transformation of the displacement calculated by a Fast Fourier Transformation technique

137 in (Boyer and López-Corona, 2009) which we propose is analogous to groundwater flow. Three 138 values of $\beta=\{2,3,5\}$ were considered, corresponding to disordered, complex and ordered media.

139 Then all the 50 power spectra were averaged and fitted by an inverse power law $S(f) \sim f-\lambda$. 
140 White noise correspond to a $\lambda \approx 0$, pink to a $\lambda \approx 1$, and brown to a $\lambda \approx 2$.

141 These results show that the emergence of pink noise for a traveling agent in a

142 heterogeneous medium depends on its degree of heterogeneity. Thus this dynamical behavior

143 may naturally arise from the motion of agents in a complex medium. The agents may be

144 frougivorous monkeys, and the complex medium a rain forest; or the agents may be water

145 particles and the medium an aquifer with a complex geology. Our results suggest that $1 / \mathrm{f}$ noise

146 may be a fingerprint of a statistical phase transition from randomness (low correlation associated

147 with white noise), to predictability (high correlation associated to brown noise) an idea suggested

148 to us by Alejandro Frank (Personal communication, 2011) and discussed in (Fossion et al. 2010).

$149 \quad 3$ Study Case

150 As part of an academic collaboration between German Karlsruhe Institute of Technology

151 (KIT) and Mexico's National University (UNAM), pumping tests were performed on a set of

152 urban well in the metropolitan zone of the San Luis Potosi city in Mexico (ZMSLP), which

153 hydrogeology is described in (Martinez et al. 2010, and Martinez et al. 2011).

154 The metropolitan area is located approximately 400 kilometers northwest of Mexico City.

155 It lies in the San Luis Potosi valley in the west-centre of the state of the same name at an altitude

156 between 1,850 and 1,900 meters above sea level. The area is flanked by the hills of Sierra San

157 Miguelito to the west and Cerro San Pedro to the east; the hills have an altitude of more than

1582300 meters. The climate is semi-arid with an average rainfall of $356 \mathrm{~mm}$ between 1989 and

1592006 , an average annual temperature of $17.68^{\circ} \mathrm{C}$, and average annual potential evaporation of

160 approximately $2,000 \mathrm{~mm}$. The San Luis Potosi aquifer system underlies much of the surface

161 endorheic basin. It consists of a shallow aquifer and a deep one, separated by a lens of fine

162 material that permits very little interaction. The shallow aquifer is recharged by rainfall in the 163 valley and the Sierra San Miguelito foothills, as well as by leaks from the urban water system.

164 The deep aquifer is recharged in the Sierra San Miguelito and beyond. The $300 \mathrm{Km} 2$ of shallow 
165 aquifer underlies the urban zone and its periphery. The thickness of the aquifer is within a range

166 estimated at four to 60 meters, while the depth of the phreatic level has been reported in general

167 terms at between five and 30 meters. The less deep levels are to be found within the urban zone

168 and they deepen towards the east and northeast in the area of peripheral farmland, following the

169 direction of the flow. The deep aquifer covers about $1980 \mathrm{Km} 2$ and underlies the municipalities

170 of San Luis Potosi and Soledad de G. Sanchez, as well as part of Cerro San Pedro, Mexquitic and

171 Zaragoza. The aquifer consists of granular material and fractured volcanic rock, and is confined

172 over most of the flat part of the basin. Usually, wells tapping this aquifer terminate at a depth of

$173350-450$ meters and exceptionally at 700 meters.

174 The time series from three pumping well tests, in the shallow aquifer, were analyzed. A

175 pumping test is conducted to evaluate an aquifer by "stimulating" the aquifer through constant 176 pumping, and observing the aquifer's response (drawdown) in observation wells. The power 177 spectrum from all tests shows that there are two statistical regimes (Figure 2). The first regime is 178 characterized by time periods from $101 \mathrm{~s}$ to $103 \mathrm{~s}$ and a $1 / \mathrm{f}$ noise statistical behavior, and the 179 second one with periods of seconds or less and a white noise type of signal.

180

181 182

Figure 2 Power spectra for three pumping tests in the aquifer of San Luis Potosi City in Mexico. Drawdown data was acquired in a 3 seconds intervals basis, with a total of 1800 measurements. There are two statistical regimes $101 \mathrm{~s}$ to $103 \mathrm{~s}$ with a $1 / \mathrm{f}$ noise statistical behavior, and the second one with periods of seconds or less and a white noise type of signal. 
187 impossible to find a unique set of parameters to represent reality correctly. Stresses and boundary

188 conditions are also uncertain; the extraction of water through wells and vertical recharge due to

189 rain are not known exactly and they must be provided to the model; lateral boundaries are often

190 virtual boundaries and water exchange through them is usually uncertain. Even model structure

191 can be uncertain because a mathematical model is an approximation of reality and thus some

192 physical processes are not completely known or partially represented (Neuman, 2003). In fact,

193 the problem of characterizing subsurface heterogeneity has been one of the biggest obstacles in

194 constructing realistic models of groundwater flow (Fleckenstein et al., 2006). Koltermann and

195 Gorelick (1996) and De Marsily et al. (1998) present a good review on the subject.

196 Prediction with classical deterministic process models is constrained by several

197 mathematical limitations. For one side, there is measurement error, non-linearity and sensitivity

198 to boundary conditions (chaos) and on the other side we most face model error and inaccessible

199 or uncertain parameters and variables (Little and Bloomfield, 2010). For these reasons,

200 systematic oversimplifications in groundwater problems have been commonly made, under the

201 assumption that if the most important processes are identified, groundwater flow may be

202 sufficiently characterized.

203 On the other hand, Kirchner and coworkers (2000) found that long-term, time series

204 measurements of chloride, a natural passive tracer, in runoff in catchments exhibits a 1/f

205 dynamics and later (Scher et al. 2002) gave a physical model to explain these founding in terms 206 of CTRW.

207 Significant deviations from standard solutions have been observed in pumping tests 208 (Raghavan, 2004). Moreover, it has been reported that $1 / \mathrm{f}$ dynamics is observed in time series of 209 pumping test (Zhongwei and You-Kuan, 2007) and we showed evidence that support their 210 findings. One approach to deal with this anomalous behavior has been to formulate the 211 groundwater flow problem in the continuous time random walk (CTRW) framework (Cortis and 
212 Knubdy, 2006). Alternatively we propose a traveling agent model for groundwater flow. The

213 model proposed is an analogy of a previous one presented by (Boyer and López-Corona, 2009)

214 which was used to construct time series for agent's mean-displacement. In agreement with field

215 results, the model generates $1 / \mathrm{f}$ dynamics when the ambient where the agents move is complex.

216 For this type of medium, the step length follows a power law distribution $\mathrm{P}(1) \sim 1$-a with $\mathrm{a} \approx 2$; the

217 waiting time distribution follows a power law $y(t) \sim t-d$ with $d=2$ and the mean displacement a

218 power law (R2) Tg with g 1.2 (Boyer and López-Corona, 2009; López-Corona, 2007). If the

219 process was a CTRW then the following relationship should hold $\mathrm{g}=2+\mathrm{d}-\mathrm{a}$ and a value of $\mathrm{g}=2$

220 would be expected (Klafter et al. 1995). This suggests that groundwater flow is even more

221 complex than a CTRW, which in fact also occurs in spider monkeys foraging process for which

$222 \mathrm{~g}=1.7$ (Ramos- Fernández et al. 2004). In this sense, the model proposed could be a forward step

223 in the study of groundwater flow complexity.

224 Another advantage of the traveling agent model for explaining the emergence of $1 / \mathrm{f}$ is that

225 we may identify in which type of hydrogeological medium this kind of dynamic behavior is

226 observed. We proved that pink noise is present when the environment heterogeneities in which

227 the agents are moving are distributed as a power law with a scaling exponent of $\beta=3$,

228 corresponding to a complex medium. At this respect, Labat (2011) has pointed out that the

229 complex characteristics of karstic aquifers make their exploitation more complicated than other

230 porous or fractured aquifers. These types of aquifers are spatially complex (as our $\mathrm{a}=3$ medium)

231 groundwater systems characterized by an inherent temporal non-stationarity and nonlinearity of

232 their hydrological response.

233 (Eliazar and Klafter 2009a, 2009b) have proven that the 1/f statistical dynamics is 234 originated by the superposition of an infinite number of stochastic processes. This suggests that 235 for complex media (as karstic or rock fractured aquifer) no groundwater modeling simplification 
236 is valid. This ambient induces a $1 / \mathrm{f}$ noise and an infinite number of stochastic processes are in

237 play. Therefore, the assumption that groundwater flow may be sufficiently characterized if the

238 most important processes are identified is no longer valid.

239 Even more, the results may be interpreted also from a physical stand point; the observable

240 macroscopic behavior of a hydrogeological system at a given location is the result of the

241 superposition of different physical processes at different scales, such as: diurnal barometric

242 variations that affect groundwater levels, temporal fluctuations in recharge rates, moon's

243 gravitational effects over the aquifer, tide variations in coastal aquifers, variations in the income

244 flow from rivers and discharge through base flow, temporal increase on total stress due to trains,

245 the effect of extraordinary recharge events provoked by an hurricane presence, and the regime of

246 operation of wells in the area. In Labat et. al. (2011) it has been proved, using DFA analysis, that

247 in Karstic stream flow fluctuations there are three distinct temporal scale ranges: from $1 \mathrm{~h}$ to

248 around $100 \mathrm{~h}$, from around $100 \mathrm{~h}$ up to 1 year and scales larger to 1 year. Fluctuations in flow

249 show a clearly anti-correlated behavior on time scales above 1 year, with a slope around 0.3

250 corresponding to white noise. In the intermediate regime from a few days up to 1 year, a positive

251 Hurst effect is observed, with a slope around 0.8 (almost a 1/f noise) as expected. On time scales

252 below the crossover at a few days, the scaling behavior is highly non-stationary and corresponds

253 to a random walk with positively correlated steps (with a slope around 1.75, near a Brown noise

254 type). The authors explain these findings from a hydrogeological point of view. The first temporal

255 scale, 1 to $100 \mathrm{~h}$, is interpreted as the rapid response of the aquifer (associated with the main

256 drain in the karstic system) to the rainfall; the second temporal scale, $100 \mathrm{~h}$ to 1 year, is the global

257 response of the aquifer to rainfall input including the temporal structure of the peak flow; the

258 third temporal scale, larger than 1 year, corresponds to the annual response of rainfall input

259 including the regulation of the discharge via annex systems in the saturated zone. It has also been 260 suggested that an explanation for the scale invariance of groundwater levels involve the system 
261 response to constantly changing driving inputs and boundary conditions, including boundaries

262 imposed by management regimes, (Little and Bloomfield, 2010). In this way, the $1 / \mathrm{f}$ power

263 spectrum observed in groundwater time series may be originated by both, complexity of the

264 geological medium and the presence of complex external factors (as time dependent boundary

265 conditions).

266 Given this, either we accept that these types of complex groundwater systems are not

267 suitable of being modeled or we learn to deal with this infinite superposition of stochastic

268 processes. Once groundwater flow is modeled on a traveling agent framework, we propose to

269 describe it as a spatially extended game. Using this approach we have been able to deduce a set of

270 partial differential equations starting from the discrete description of the model (the details of the

271 derivation are presented as Electronic Supplementary Material). The probability of finding an

272 agent (water particle) in the position (x,y) at the time t obeys

273

$$
\partial_{\mathrm{t}} \mathrm{P}(\mathrm{x}, \mathrm{y}, \mathrm{t})=\operatorname{div}[\mathrm{e}(\mathrm{x}, \mathrm{y}, \mathrm{t}) \nabla \mathrm{P}],
$$

274

where $\mathrm{e}(\mathrm{x}, \mathrm{y}, \mathrm{t})$ is the strategy (micro-physics of the flow process) that the agent in $(\mathrm{x}, \mathrm{y})$

275 plays at time t. The strategy in turn obeys the equation

$276 \partial_{\mathrm{t}} \mathrm{e}(\mathrm{x}, \mathrm{y}, \mathrm{t})=-\operatorname{div}\left[\mathrm{D}_{1}(\mathrm{x}, \mathrm{y}, \mathrm{t}) \mathrm{e}(\mathrm{x}, \mathrm{y}, \mathrm{t})\right]+\nabla^{2}\left[\mathrm{D}_{2}(\mathrm{x}, \mathrm{y}, \mathrm{t}) \mathrm{e}(\mathrm{x}, \mathrm{y}, \mathrm{t})\right]$

277 While in continuous time random walk approaches few parameters suffice to describe a

278 complex system, Eqs (1) and (2) introduce field (x,y) dependent diffusion

279 and drift coefficients, and thus represent a quite complex approach. At this respect, Godec and

280 Metzler (2013) has provied an exact expression for the diffusion coefficient in anomalous

281 diffusion process modeled by Lévy walks under linear response regime.

282 If you take the simple case when $e(x, y, t)$ is a constant (considering that the porous

283 medium es relative constant in the observation time scale, and it is sufficiently homogeneous and

284 isotropic, which by the way are common assumptions in hydrogeology), then you recover the 
285 classical groundwater flow equation $S_{s} \partial h / \partial t=k \nabla^{2} h$. Our equations then satisfy the

286 correspondence principle since they recover classical formulation and establish the ground for

287 new insights of groundwater flow process, other porous media transport phenomena and even in

288 Game Theory.

289 Typically, a system is considered complex when it is constituted from a large number of

290 subsystems that interact strong enough, but there is another source of complexity that has been

291 widely ignored. A system is also complex when the systems itself changes over time in the same

292 scale of its dynamics, which is be the case in some karstic aquifers. This second source of

293 complexity is taken into account directly in our equations making a contribution in this respect

294 and might have some important interpretation in Game Theory.

295 Finally, most interesting, using the traveling agent model described in the method section,

296 we proposed (Lopez-Corona et al. 2013) that $1 / \mathrm{f}$ noise is a fingerprint of a statistical phase

297 transition, form randomness (disorder - white noise) to predictability (order - brown noise). In

298 this context, one may interpret Labat et al., (2011) results as follows: first temporal scale (from 1

299 to $100 \mathrm{~h}$ ) represents a the rapid response of the aquifer and should be dominated by random

300 processes (white noise); the second (100 $\mathrm{h}$ to 1 year) is the global response of the aquifer to

301 rainfall input including the temporal structure of the peak flow one may be interpreted as a

302 complex (with multiple spatio and temporal scales included) process (1/f noise); and as the third

303 correspond to mean (1 year or more) response is a more predictable process (brown noise). We

304 have then a transition from randomness to predictability consistent with power spectra exponent

305 values. In this way, the results of Labat et al. (2011) is only one example of a universal statistical

306 kind of phase transition.

$307 \quad$ Acknowledgements

308 This work was supported by CONACyT scholarship within the graduate program in Earth 
310 Tomas González are gratefully acknowledged.

\section{$311 \quad$ References}

312

313

314

315

316

317

318

319

320

321

322

323

324

325

326

327

328

329

330

331

332

333

334

335

336

337

338

339

340

341

342

343

344

345

346

347

348

349

350

351

352

353

354
Accardi L. and M. Regoli (2000), Non-locality and quantum theory: new experimental evidence, arXiv.org/abs/quant-ph/0007019v1

Accardi L. and M. Regoli (2001), The EPR correlations and the chameleon effect, arXiv.org/abs/quant-ph/0110086v1

Atkinson, R., C. Rhodes, D. MacDonald and R. Anderson (2002), Scale-free dynamics in the movement patterns of jackals, Oikos 98 134-40.

Austin, D., W. Bowen and J. McMillan (2004), Intraspecific variation in movement patterns: modeling individual behaviour in a large marine predator, Oikos 10515 30

Bak, P.C. and K. Chen (1991), Self-organized criticality, Scientific American, "SelfOrganized Criticality." Scientific American 264(1), 46-54.

Bak, P.C. and K. Tang (1989), Earthquakes as a self-organized critical phenomenon, Geophys. 94, No. B 11, 15,635-15,637.

Bak, P.C., K. Tang and K. Weisenfeld (1987), Self-organized criticality: an explaination of 1/f noise, Phys. Rev. Lett. 59 (4), 381-384

Bohigas, O. M. Giannoni and C. Schmit (1984), Characterization of chaotic quantum spectra and universality of level fluctuation laws, PRL 52:1,1-4.

Boyer, D. and O. López-Corona (2009), Self-organization scaling and collapse in a coupled automaton model of foragers, J. Phys. A: Math. Theor. 42

Boyer, D., O. Miramontes and H. Larralde (2009), Lévy-like behaviour in deterministic models of intelligent agents exploring heterogeneous environments, J. Phys. A: Math. Theor. 42

Boyer, D., G. Ramos-Fernández, O. Miramontes, J.L. Mateos, G. Cocho, H. Larralde, H. Ramos. and F. Rojas (2006), Scale-free foraging by primates emerges from their interaction with a complex environment, Proc. R. Soc. B 273, 1743-50

Buhl, J., D. Sumpter, Couzin D., Hale J.J., Despland E., Miller E. R., S. J. Simpson (2006). From disorder to order in marching locusts, Science, 312(5778), 14021406.

Cavagna, A., A. Cimarelli, I. Giardina, G. Parisi, R. Santagati, F. Stefanini, M. Viale (2009), Scale-free correlations in bird flocks, arXiv 0911.4393.

Cortis, A. and C. Knudby (2006), A continuous time random walk approach to transient flow in heterogeneous porous media. Water Resources Research 42, W10201.

De Marsily, G., F. Delay, V. Teles and M.T. Schafmeister (1998), Some current methods to represent the heterogeneity of natural media in hydrogeology. Hydrogeology Journal Vol. $6: 115-130$

Dentz, M., Gouze, P., Russian, A., Dweik, J., \& Delay, F. (2012). Diffusion and trapping in heterogeneous media: An inhomogeneous continuous time random walk approach. Advances in Water Resources, 49, 13-22.

Downey, A. (2012), Think Complexity. O’Reilly Media. pp. 79. ISBN 978-1-4493-14637.

Duffy, C.J., and L.W. Gelhar (1985), A frequency domain approach to water quality modeling in groundwater: Theory. Water Resources Research 21, no. 8: 1175-1184. 
Duffy, C.J., and S. Al-Hassan (1988). The time and frequency response of tracer experiments. Journal of Hydrology 97, no. 1-2, 59-73.

Eady, E. T. (1951), The Quantitative Theory of Cyclone Development. In: Compendium of 387 Meteorology. T. F. Malone (Ed.), American Meteorological Society, pages 464-469.

Eliazar, I. and J. Klafter (2009a), A unified and universal explanation for Lévy laws and 1/f noises, PNAS Vol. 106 (30) 12251 - 12254.

Eliazar, I. and J. Klafter (2009b) Universal Generation of Statistical Self-Similarity: A Randomized Central Limit Theorem, Phys. Rev. Lett. 103

Eliazar, I., \& Klafter, J. (2010). Universal generation of 1/f noises. Physical Review E, 82(2), 021109.

Faleiro, E., U. Kuhl, R. Molina, L. Muñoz, A. Relanyo and J. Retamosa (2006), Power spectrum analysis of experimental sinai quantum billiards, PLA 358, 251-255.

Fleckenstein, J., R. Niswonger and G. Fogg (2006), River-Aquifer Interactions, Geologic Heterogeneity, and Low-Flow Management. Groundwater, 44(6) 837-852

Fossion R., E. Landa, P. Stransky, V. Velazquez, J.C. Lopez Vieyra, I. Garduno, D. Garcia, and A. Frank (2010). Scale invariance as a symmetry in physical and biological systems: listening to photons, bubbles and heartbeats, AIP Conf. Proc. 1323, 74, DOI:10.1063/1.3537868

Gelhar, L.W. (1974) Stochastic analysis of phreatic aquifer, Water Resources Research 10, no. 3: 539-545.

Gisin N. (2007), How come the Correlations?, arXiv:quant-ph/0503007v1.

Godec and Metzler, (2013) Linear response, fluctuation-dissipation, and finite-system-size effects in superdiffusion, Phys Rev E 88, 012116

Goldberger, A. (2002), Fractal dynamics in physiology: alterations with disease and aging, PNAS 99(1), 2466-2472

Haq, R., A. Pandey and O. Bohigas (1982), Fluctuation properties of nuclear energy levels do theory and experiment, agree, PRL 48(16), 1086-1089.

Hardy, L. (2001), Quantum Theory from Five Reasonable Axioms, http://arXiv.org/abs/quant-ph/0101012v4

Herrera, G. and G. Pinder (2011), Notes on Stochastic Simulation (unpublished)

Jianting Z., M. Young and J. Osterberg (2012), Impacts of riparian zone plant water use on temporal scaling of groundwater systems, Hydrol. Process. 26, 1352-1360

Keilis-Botok, V. (1990). The lithosphere of the Earth as a non-linear system with implications for earthquakes prediction, Rev. Geophys. 28(1),19-34.

Khrennikov A. (2004). Växjö interpretation-2003: realism of contexts, arXiv.org/abs/quant-ph/0401072v1.

Khrennikov, A. (2008). Bell-Boole inequality: nonlocality or probabilistic incompatibility of random variables?. Entropy, 10(2), 19-32.

Khrennikov A. (2008b). Probabilistic foundations of quantum mechanics and quantum information. arXiv.org/abs/quant-ph/0309066v1.

Khrennikov, A. (2009a). Contextual approach to quantum formalism (Vol. 160). Springer, 353.

Khrennikov, A. (2011). Quantum-like model of processing of information in the brain based on classical electromagnetic field. Biosystems, 105(3), 250-262.

Kirchner, J. W., X. Feng and C. Neal C. (2000), Fractal stream chemistry and its implications for contaminant transport in catchments, Nature, 403, 524-527.

Klafter, J., Blumen, A., \& Shlesinger, M. F. (1987). Stochastic pathway to anomalous 
diffusion. Physical Review A, 35(7), 3081-3085.

Klafter J., G. Zumofen and M.F. Shlesinger (1995) Lévy description of anomalous diffusion in dynamical systems in LEVYY FLIGHTS AND RELATED TOPICS IN PHYSICS, Lecture Notes in Physics, Volume 450/1995, 196-215, DOI: 10.1007/3540-59222-9 35

Koltermann, D. and S. Gorelick (1996), Heterogeneity in Sedimentary Deposits: A Review of Structure-Imitating, Process-Imitating, and Descriptive Approaches. WATER RESOURCES RESEARCH, 32(9), P. 2617-2658, doi:10.1029/96WR00025.

Kracklauer A. (2001). Is "entanglement" always entangled?. arXiv.org/abs/quant$\mathrm{ph} / 0108057 \mathrm{v} 2$

Labat, D., J. Masbou, E. Beaulieu, and A. Mangin (2011), Scaling behavior of the fluctuations in stream flow at the outlet of karstic watersheds, France. Journal of Hydrology 410, 162-168.

Little M. and J. Bloomfield (2010), Robust evidence for random fractal scaling of groundwater levels in unconfined aquifers, ournal of Hydrology 393, 362-369

Lomholt, M. A., Tal, K., Metzler, R., \& Joseph, K. (2008). Lévy strategies in intermittent search processes are advantageous. Proceedings of the National Academy of Sciences, 105(32), 11055-11059.

López-Corona O. (2007), Modelos estadísticos de difusión en ambientes desordenados. Aplicación al forrajeo y dispérsión de semillas en selvas. Bachelor degree dissertation.

López-Corona, O., P. Padilla, O. Escolero, A. Frank, R. Fossion (2013), Lévy flights, 1/f noise and self organized criticality in a traveling agent model. Journal of Modern Physics Vol 4, No 3, 337-343.

Maréchal, J.C., M.P. Sarma, S. Ahmed, and P. Lachassagne. (2002), Establishment of earth tide effect on water-level fluctuations in an unconfined hard rock aquifer using spectral analysis. Current Science 83, no. 1: 61-64.

Martínez, S., O. Escolero and S. Kralish (2010), Water Management in San Luis Potosi Metropolitan Area, México. International Journal of Water Resources Development. Vol. 26, Num. 3, 459-475.

Martinez, S., O. Escolero and L. Wolf (2011), Total urban water cycle models in semiarid environments - Quantitative scenario analysis at the area of San Luis Potosi, Mexico. Water Resources Management. Vol. 25, Num. 1, 239-263.

Miller, S.L., W.M. Miller and P.J. McWhorter (1993), Extremal dynamics: A unifying and activated processes physical explanation of fractals, i/f noise, J. Appl. Phys. 73 (6), $2617-2628$.

Miramontes, O., D. Boyer and F. Bartumeus (2012), The Effects of Spatially Heterogeneous Prey Distributions on Detection Patterns in Foraging Seabirds. PLoS ONE 7(4): e34317. doi:10.1371/journal.pone.0034317

Montroll, EW, and Shlesinger Michael F (1982). On 1/f noise and other distributions with long tails. Proceedings of the National Academy of Sciences. 72(10), 3380-3383

Neuman S.P. (2003) Maximum likelihood Bayesian averaging of uncertain model predictions. Stochast Environ Res Risk Assess 17(5):291-305. doi:10.1007/s00477-003-0151-7.

Niemann M., Szendro I. and Kantz H. (2013). 1/ $\mathrm{f}^{\wedge}$ beta noise in a model for weak ergodicity breaking. arxiv.org/abs/1002.0993

Park, C.H., Beyer, C., Bauer, S., Kolditz, O. (2008): An efficient method of random walk particle tracking: accuracy and resolution. Geosciences Journal, 12(3), 285-297. 
452

453

454

455

456

457

458

459

460

461

462

463

464

465

466

467

468

469

470

471

472

473

474

475

476

477

478

479

480

481

482

483

484

485

486

487

488

489

490

491

492

493

494

495

496

497

498

499

500
Press, W.H. (1978), Flicker Noises in Astronomy and Elsewhere, Comments Astrophys. 7, 103-119.

Raghavan, R. (2004), A review of applications to constrain pumping test responses to improve on geological description and uncertainty. Rev. Geophys, 42, RG4001, doi:10.1029/2003RG000142.

Ramos-Fernández, G., J.L. Mateos, O. Miramontes, G. Cocho, H. Larralde and B. AyalaOrozco (2004), Lévy walk patterns in the foraging movements of spider monkeys (Ateles geoffroyi), Behav. Ecol. Sociobiol. 55, 223-30

Relanyo, A., J. Gómez, R. Molina, J. Retamosa and E. Faleiro (2002), Quantum chaos and 1 over f noise, PRL 89:244102

Sauquet, E., M.H. Ramos, L. Chapel, and P. Bernardara (2008), Streamflow scaling properties: Investigating characteristic scale from different statistical approaches. Hydrological Processes 22, no. 17: 3462-3475.

Seuront, L., A. Duponchel and C. Chapperon (2007), Heavy-tailed distributions in the intermittent motion behaviour of the intertidal gastropod Littorina littorea. Physica A 385, 573-82

Scher, H., Cortis, A., \& Berkowitz, B. (2002, December). A Unified Framework for Anomalous Transport in Geological Formations. In AGU Fall Meeting Abstracts (Vol. 1, p. 04).

Schilling, K.E., J.A. Palmer, E.A. Bettis III, P. Jacobson, R.C. Schultz, and T.M. Isenhart (2009), Vertical distribution of total carbon, nitrogen and phosphorus in riparian soils of Walnut Creek, southern Iowa (USA). Catena 77, no. 3: 266-273.

Shlesinger, M. F., Klafter, J., \& Wong, Y. M. (1982). Random walks with infinite spatial and temporal moments. Journal of Statistical Physics, 27(3), 499-512.

Shih, D., C. Lee, K. Chiou, and S. Tsai (2000), Spectral analysis of tidal fluctuations in ground water level. Journal of the American Water Resources Association 36, no. 5: 1087-1099

Simon B., S. Simon, F. Gori, M. Santarsiero, R. Borghi, N. Mukunda. and R. Simon (2010). Nonquantum Entanglement Resolves a Basic Issue in Polarization. Optics Phys. Rev.Lett. 104 (2).

Sims, D.W., E.J. Southal, N.E. Humphries, G.C. Hays, C.J.A. Bradshaw, J.W. Pitchford, A. James, M.Z. Ahmed, A.S. Brierley, M.A. Hindel, D. Morritt, M.K. Musyl, D. Righton, E.L.C. Shepard, V.J. Wearmouth, R.P. Wilson, M.J. Witt \& J.D. Metcalfe (2008). Scaling laws of marine predator search behaviour. Nature 451, 1098-102.

Sornette, A. and D. Sornette (1989), Self-organized criticality and earthquakes, Europhys. Lett. 9, 197-202.

Sornette, D., P. Davy and A. Sornette (1990), Structuration of the lithosphere in plate tectonics as a Self-organized criticality Phenomenon, J. Geophys. Res. 95, no. B11,17353-61.

Tessier, Y., S. Lovejoy, P. Hubert, D. Schertzer, and S. Pecknold (1996), Multifractal analysis and modeling of rainfall and NGWA.org river flows and scaling, causal transfer functions. Journal of Geophysical Research 101, no. D21: 26427-26440.

Tranouez, P., C. Bertelle and D. Olivier (2001), Changing the level of description of a fluid flow in a agent-based simulation. In ESS 2001 Conference, Marseilles (France).

Viswanathan, G.M., S.V. Buldyrev, S. Havlin, M. da Luz, E. Raposo and H. Stanley (1999). Optimizing the success of random searches. Nature 401 911-14

Zhongwei, L., Z. You-Kuan (2007), Quantifying fractal dynamics of groundwater systems with detrended fluctuation analysis, Journal of Hydrology, 336 139-146. 
501 Zhang, Y.-K., and Z. Li (2005), Temporal scaling of hydraulic head fluctuations: nonstationary spectral analyses and numerical simulations, Water Resour. Res., 41(7), W07031

Zhang, Y.-K., and Z. Li (2006). Effect of temporally correlated recharge on fluctuations of groundwater levels. Water Resources Research 42(10): W10412

Zhang, Y.K., K. Schilling (2004), Temporal scaling of hydraulic head and river base flow and its implication for groundwater recharge. Water Resources Research 40(3) W03504. 


\section{Table 1 (on next page)}

Relation between media homogeneity coefficient $\beta$, type of medium, agent motion, and the noise type observed. 


\begin{tabular}{ccrc} 
Homogeneity & Medium type & Agent motion type & Displacement noise type \\
\hline$\beta=2$ & Inhomogenous & Random Confined & White \\
& Disordered & & Uncorrelated \\
$\beta=3$ & Complex & Lévy & Pink (1/f) \\
& Transition point & Fractal & Transition point \\
$\beta=5$ & Homogeneous & Brownian & Brown \\
& Ordered & & Highly correlated
\end{tabular}




\section{Figure 1}

Power spectra for traveling agents with three values of homogeneity.

First column $\beta=2$, the medium is very inhomogeneous (disordered) and the signal is a white noise. Second column $\beta=3$, the medium is complex and the signal is a pink noise. Third column 5 , the medium is very homogeneous (ordered) and the signal is a brown noise. Power Spectrum is taken as $S(f) \equiv R(f) R(-f)$, where $R(f)$ is the Fourier transformation of the displacement calculated by a Fast Fourier Transformation technique.
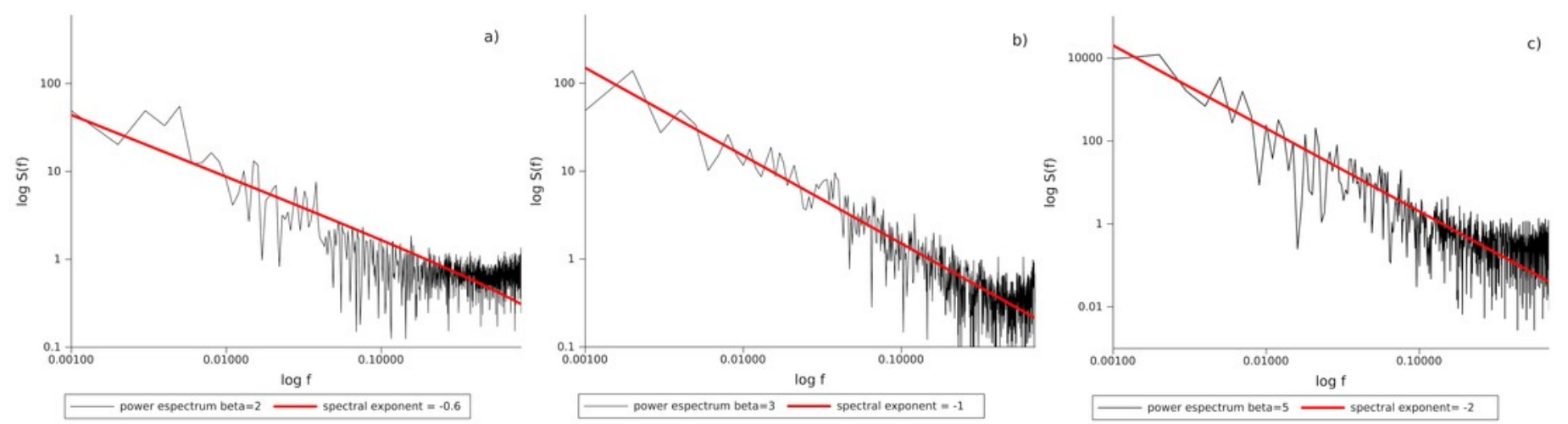


\section{Figure 2}

Power spectra for three pumping tests in the aquifer of San Luis Potosi City in Mexico.

Drawdown data was acquired in a 3 seconds intervals basis, with a total of 1800 measurements. There are two statistical regimes $101 \mathrm{~s}$ to $103 \mathrm{~s}$ with a $1 / \mathrm{f}$ noise statistical behavior, and the second one with periods of seconds or less and a white noise type of signal.
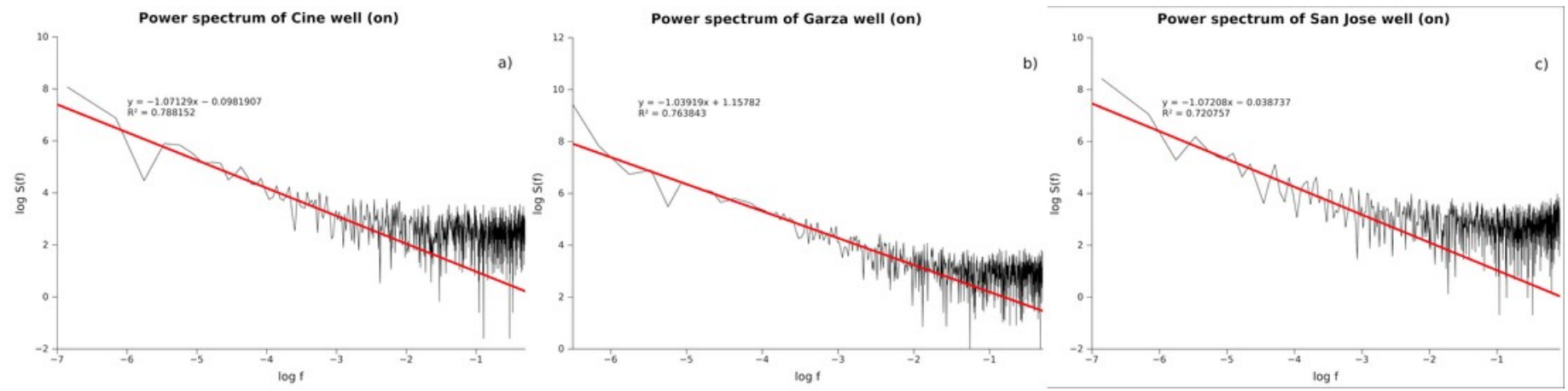